\title{
Unified model for quasar absorption line systems
}

\author{
S. Das, P. Khare, and A. Samantray \\ Physics Department, Utkal University, Bhubaneswar, 751004, India \\ Received 19 January 2001 / Accepted 2 May 2001

\begin{abstract}
We propose a three component model consisting of minihalos and galactic halos with embedded thin discs for absorbers producing all the observed classes of intervening quasar absorption line systems. We show that this model, based on CDM cosmology, can explain most of the observed statistical distributions of various types of absorption systems. Use of the Schechter luminosity function for absorbers, on the other hand, is consistent with the observations only if the number of galaxies was larger in the past and reduced with time due to mergers. A strong chemical evolution in the halos of galaxies is indicated by the observed properties of CIV lines. We discuss our results in the light of recent observations of the absorption line systems.
\end{abstract}

Key words. quasars - absorption lines - galaxies - abundances, galaxies - halos

\section{Introduction}

Quasar absorption lines provide unique, sensitive and direct probes of the gaseous content of galaxies and intergalactic medium (IGM) over look back times comparable to a Hubble time. Studies of these lines promise to provide insight into the processes that regulate the evolution of the IGM and the galaxies and the mechanisms that determine the physical conditions in discs and in clouds embedded in the halo. In a typical quasar spectra there are several populations of absorption lines and it has become apparent that these different populations are likely the absorption signatures of different regions and conditions within the galaxies and the IGM.

Narrow, heavy element absorption systems (HEASs) in the quasar spectra are believed to be produced by gas in the halos of intervening galaxies. This was demonstrated by the high success rate of detection of emission at the redshift of the intermediate redshift $(z \sim 0.5-0.8) \mathrm{MgII}$ absorption systems (Bergeron 1988; Steidel 1993). High resolution studies of these heavy element lines have revealed that the lines break up into several distinct components which are most likely produced by clouds in the halos of the absorbing galaxies (Srianand \& Khare 1994). Most of the Lyman limit systems (LLSs), which have a neutral hydrogen column density $>2 \times 10^{17} \mathrm{~cm}^{-2}$, are associated with the HEASs.

Damped Ly $\alpha$ systems (DLASs) are produced by condensations of neutral gas having neutral hydrogen column density $N_{\mathrm{HI}}>10^{20.3} \mathrm{~cm}^{-2}$ and are easily detectable in the spectra of background quasars (Wolfe et al. 1986).

Send offprint requests to: P. Khare, e-mail: khare@iopb.res.in
These are associated with metal lines of low ionization and often high ionized species as well. Although the exact nature of the DLASs has yet to be understood, it is generally believed that they are produced by the progenitors of present day galaxies. This conclusion is based on the following facts (i) the mass contained in these systems at redshifts $>2$ seems to be comparable to the stellar mass at present and (ii) the kinematics of the gas in DLASs seems to indicate their origin in rotating discs. This view has been questioned recently by several authors in the light of some of the new data on these systems. We will discuss these later on in this paper.

Lanzetta et al. (1995) and Chen et al. (1998), from a comparison of galaxy and absorber redshifts, showed that a dominant fraction of low redshift $(z<1)$ Lyman alpha absorption systems (LAASs) arises in extended halos of galaxies. This was also corroborated by the discovery of heavy elements (Cowie et al. 1995) at redshifts around 3 and of clustering in these systems at redshifts between 1.7 and 3.8 (Srianand \& Khare 1994). Recently Chen et al. (2000) considered the statistical properties of the high redshift galaxies in the Hubble deep field and the statistical properties of Ly $\alpha$ absorption systems. They conclude that at least $50 \%$ and possibly as high as $100 \%$ of observed LAASs arise in extended envelopes of galaxies at $z>2$. It thus appears that a substantial fraction of the LAASs may be produced by the outer parts of galactic halos, rather than in the IGM as believed earlier (Sargent et al. 1980).

During the past few years, there has been a maturing of attempts to understand the nature of these intervening populations, evolving from rather simple idealized models (e.g. pressure confined clouds, gravitationally confined "minihalos", freely expanding clouds etc.) to sophisticated 
numerical simulations that are able to reproduce many of the features of the absorption spectra in the context of the evolution of structure (Chiba \& Nath 1997; Das \& Khare 1999; Charlton \& Churchill 1996; Kauffmann 1996; hereafter K96, Gnedin \& Ostriker 1996 etc.). Das \& Khare (1999) have considered a two component (minihalos and galactic halos) CDM model to understand the LAASs and associated CIV absorption lines. K96 considered the formation of discs in CDM models and showed that the observed properties of the DLASs can be accounted for by these models. This pure disc model for DLASs was questioned by Prochaska \& Wolfe (1997) on the basis of the kinematic structure of the DLASs. Subsequently, Jedamzik \& Prochaska (1998) have shown that only a finely tuned disc model within the framework of CDM could be made marginally consistent with the damped Ly $\alpha$ observations.

The purpose of this paper is to explore a consistent model of minihalos and of galaxies which are assumed to have spherical and photo ionized gaseous clouds embedded in their halos with a disc at the centre, to better understand the nature of the LAASs, HEASs and DLASs in a unified manner. In Sect. 2, we describe the basic model with the underlying hypothesis. We discuss the orientation of the discs inside the halo in Sect. 3. We explore the field of parameters and constrain these parameters using observations in Sect. 4. We present the results in Sects. 5 and 6 before drawing our conclusions in Sect. 7. A discussion of the results in view of some of the recent observations is presented in Sect. 8 .

\section{The basic model}

The two component model was recently considered by Das \& Khare (1999) to understand the observations of Lyman $\alpha$ forest lines and their accompanying $\mathrm{C} \mathrm{IV} \mathrm{lines} \mathrm{in} \mathrm{the}$ framework of the hierarchical structure formation model in CDM cosmology. This model consists of minihalos and galactic halos. These are dark matter halos having $5 \%$ of their mass in the form of baryons. The Minihalos have circular velocities, $V_{\mathrm{c}}$ between 15 and $55 \mathrm{~km} \mathrm{~s}^{-1}$ and consequently have a smaller virial radius (see Eq. (2.1)). The lower limit of $15 \mathrm{~km} \mathrm{~s}^{-1}$ is roughly the value below which the perturbations in the baryonic mass are suppressed, while $55 \mathrm{~km} \mathrm{~s}^{-1}$ is roughly the value below which the photo ionization by the external UV background suppresses cooling and therefore the formation of clouds and stars. These are chemically enriched, if at all, by an earlier generation of stars.

Halos with circular velocities larger than $55 \mathrm{~km} \mathrm{~s}^{-1}$ are called galactic halos. The upper limit on $V_{\mathrm{c}}$ for galactic halos is taken to be $500 \mathrm{~km} \mathrm{~s}^{-1}$, as the results presented below do not change if the upper limit is increased above this value. The gas in these halos can cool due to higher gas densities inside (see below) and form clouds and stars. Thus these halos have clouds embedded in them which are in pressure equilibrium with the hot intercloud medium. Their number density is assumed to have the same radial dependence as the total density. Here, we extend this model to include the presence of isothermal discs inside the galactic halos which form as the gas cools, collapses and forms stars (K96). It may be noted that all the halos in our model, being gravitationally confined, are assumed to be spherical. There have been suggestions in the literature of the filamentary nature of the Ly $\alpha$ absorbers (Charlton et al. 1993). These were based upon the large transverse sizes of these absorbers $(\geq 100 \mathrm{kpc})$ obtained from the occurrence of common absorption lines in the spectra of multiple images of the lensed QSOs (Smette 1995). Similar sizes along the line of sight (los) (as would be required for the spherical absorbers) would require too low a value of the external (IGM) pressure. This is however, not a problem in our model as the galactic halos (where the lines observed by Smette (1995) at low resolution would have originated) have clouds with sizes of about a few kpc embedded in them. The common absorption lines in the images of lensed QSOs may be produced by different clouds in the same halo. Thus, the assumption of spherical halos does not violate the constraints from the lensed QSO data. We note that the lensing data indicate that if the los to one image intercepts an absorber, the probability that the los to the other image intercepting the same absorber is $>90 \%$. We will discuss this with reference to our model in Sect. 4.

Our model for the disc is based on the work of K96 according to which the gas collapses to a constant fraction of its initial virial radius to form a rotationally supported disc within the halos; the virial radius for spherical collapse model is given by

$r_{\text {vir }}=0.1 H_{0}^{-1}(1+z)^{-3 / 2} V_{\mathrm{c}}$.

Here $H_{0}$ is the Hubble constant. Using the results of an $N$-body simulation (Barnes \& Efstathiou 1987) it can be shown that for a disc/halo mass ratio of 0.1 the equilibrium disc radius is $\simeq 10 \%$ of the virial radius of the halo. A spread around this factor is expected due to possible spread in spin parameters of the dark halo (K96). We have included an additional dependence of the disc size on redshift, apart from the $(1+z)^{-3 / 2}$ dependence which is due to the $z$ dependence of $r_{\text {vir }}$. This seems to be necessary to account for the observations, as seen below. Thus the disc radius in our model is given by,

$R_{\mathrm{d}}=0.1 H_{0}^{-1}(1+z)^{a-3 / 2} V_{\mathrm{c}}$,

where $a$ is an unknown parameter whose value as well as the value of the thickness of the disc, $t$, is fixed by comparing the model results with observation of DLAs as explained below.

Following K96, we assume all the discs to have flat rotation curves and a rotational velocity equal to the circular velocity of their surrounding dark matter halos. Assuming a Kennicutt (1989) star formation law and a constant gas velocity dispersion of $6 \mathrm{~km} \mathrm{~s}^{-1}$, the gravitation stability condition gives the critical surface density as (K96)

$\Sigma_{\text {crit }}(R)=0.59 \frac{V\left(\mathrm{~km} \mathrm{~s}^{-1}\right)}{R(\mathrm{kpc})} M_{\odot} \mathrm{pc}^{-2}$, 
where $V$ is the circular velocity of the disc and $R$ is the radial distance from the centre. The surface gas density in the disc remains close to the critical density with constant infall, star formation and ejection of hot disc gas into the halo through supernovae explosions. So the surface gas density in the disc is also given by the above equation.

The total hydrogen column density contributed by the disc for an impact parameter $P$ for a face-on disc can be written as

$N_{\mathrm{HT}}^{\text {disc }}(P)=\frac{\Sigma(P)}{m}$,

$m$ being the average mass per gas particle, which we assume to be mass of the proton, $m_{\mathrm{p}}$. The average number density, $n_{\mathrm{H}}$ along a los through the disc is thus

$n_{\mathrm{H}}=\frac{N_{\mathrm{HT}}^{\mathrm{disc}}(P)}{t}$.

The total column density along a given los is the sum of the column density contributed by the disc, the column densities in all the clouds in the halo that lie along the los and the column density contributed by the hot medium along the los. We assume the number density of clouds at a radial distance $r$ from the centre of the halo, $\eta_{\mathrm{cl}}(r)$ to be

$\eta_{\mathrm{cl}}(r)=\frac{\eta_{\mathrm{cl}}(0)}{1+r^{2} / r_{\mathrm{c}}^{2}}$

$r_{\mathrm{c}}$ being the core radius. The number of clouds per unit volume at the centre of the galaxy is given by

$\eta_{\mathrm{cl}}(0)=f \frac{3}{4 \pi r_{\mathrm{cl}}^{3}(0)}$.

The value of $f$ is fixed by comparing the model results with observations, as described below.

The radial density distribution of the baryonic matter in the minihalos and in galactic halos is given by

$\rho(r)=\frac{f_{\mathrm{b}} V_{\mathrm{c}}^{2}}{4 \pi G\left(r^{2}+r_{\mathrm{c}}^{2}\right)}\left(\frac{x_{\mathrm{v}}}{x_{\mathrm{v}}-\arctan x_{\mathrm{v}}}\right)$,

where $f_{\mathrm{b}}$ is the fraction of baryonic matter in the halo, which we take to be 0.05 as mentioned above. $r_{\mathrm{c}}$ is the core radius, which is taken to be 10 and $100 \mathrm{kpc}$ for mini and galactic halos respectively and $x_{\mathrm{v}}=\frac{r_{\mathrm{vir}}}{r_{\mathrm{c}}} \simeq 15$ (Chiba \& Nath 1997).

\section{Orientation of the discs}

The orientation of the disc is an important factor to consider while determining the column density contribution of the disc, which is likely to increase due to the increased path length through an inclined disc. For the calculation of the contribution of the disc to the column density along a los with impact parameter $P$, we assume the disc to be randomly oriented with inclination $i$. At this orientation, the disc is projected on the plane of the sky onto an ellipse with area $\pi R_{\mathrm{d}}^{2} \cos i$. If the los is located at a random angle $\theta$ from the major axis of the ellipse, then the radial position along the surface of the disc where it enters the plane of the disc is given by

$R_{\text {in }}=P\left[\cos ^{2} \theta+\left(\frac{\sin \theta}{\cos i}\right)^{2}\right]^{1 / 2}$,

and the radial position along the surface of the disc where it leaves the plane of the disc is given by

$R_{\text {out }}=\left[P^{2} \cos ^{2} \theta+\left(\frac{P \sin \theta}{\cos i+t \tan i}\right)^{2}\right]^{1 / 2}$.

The column density for a particular ion along a los with impact parameter $P$, for minihalos is given by,

$N_{\text {ion }}(P)=2 \int_{P}^{R_{\mathrm{H}}} \frac{n_{\mathrm{H}}(r) f_{\text {ion }}(r) r \mathrm{~d} r}{\sqrt{r^{2}-P^{2}}}$.

Here $n_{\mathrm{H}}(r)$ is the number density of hydrogen, $R_{\mathrm{H}}$ is the radius of the halo and $f_{\text {ion }}(r)$ is the ionized fraction of the ion multiplied by $Z$, the abundance of the element being considered. $f_{\text {ion }}$ depends on the ionization parameter $\Gamma$ at the point under consideration. For galactic halos, when the los passes outside the disc the column density is given by

$N_{\text {ion }}(P)=2 \int_{P}^{R_{\mathrm{H}}} A(r) \mathrm{d} r+2 \int_{P}^{R_{\mathrm{H}}} B(r) \mathrm{d} r$.

Here, $A(r)=\frac{N_{\mathrm{H}}(r) f_{\mathrm{ion}}(r) \sigma(r) \eta_{\mathrm{cl}}(r) r}{\sqrt{r^{2}-P^{2}}}$ determines the contribution from the galactic clouds, $\sigma(r)$ being the cross section of the clouds. $N_{\mathrm{H}}(r)$ is the total hydrogen column density contributed by an individual cloud present at the radial distance $r$ from the halo centre. $B(r)=\frac{n_{\mathrm{H}}(r) f_{\text {ion }}(r) r}{\sqrt{r^{2}-P^{2}}}$ is the contribution to the column density from the intercloud medium.

When the los with an impact parameter $P$ passes through the disc, the column density for a particular ion is,

$$
\begin{gathered}
N_{\text {ion }}(P, \theta, i)=\int_{R_{\mathrm{H}}}^{\sqrt{R_{\mathrm{in}}^{2}+(t / 2)^{2}}} A(r) \mathrm{d} r+\int_{\sqrt{R_{\mathrm{out}}^{2}+(t / 2)^{2}}}^{R_{\mathrm{H}}} A(r) \mathrm{d} r \\
+\int_{R_{\mathrm{H}}}^{\sqrt{R_{\mathrm{in}}^{2}+(t / 2)^{2}}} B(r) \mathrm{d} r+\int_{\sqrt{R_{\mathrm{out}}^{2}+(t / 2)^{2}}}^{R_{\mathrm{H}}} B(r) \mathrm{d} r+\frac{f_{\mathrm{ion}} \Sigma(P)}{\mu m_{\mathrm{p}}}
\end{gathered}
$$

The first two terms give the contribution of the clouds in the halo along the los, the next two terms give the contribution from the intercloud medium in the halo while the last term gives the contribution from the disc. The disc contribution to the column density along the sides of the projected ellipse is constant, but the halo contribution changes from point to point. Here $\mu=\cos i$ and the factor of $\frac{1}{\mu}$ accounts for the increased path length through the disc due to its inclination; $\frac{f_{\text {ion }} \Sigma(P)}{m_{\mathrm{p}}}$ is the column density contributed by the disc in the face-on position. 
The ionization parameter $\Gamma$ at a radial distance $r$ from the centre is given by $\Gamma(r)=\frac{4 \pi 10^{-21} J_{21}}{c \alpha_{\mathrm{Q}} h n_{\mathrm{H}}(r)}$ where $\alpha_{\mathrm{Q}}$ is the slope of the spectrum of the UV background radiation (assumed to be a power law). We assume $\alpha_{\mathrm{Q}}=-1.5$. $J_{-21}$ is the intensity of the UV background radiation in units of $10^{-21} \mathrm{erg} \mathrm{cm}^{-2} \mathrm{~s}^{-1} \mathrm{~Hz}^{-1} \mathrm{str}^{-1}$ at 1 Rydberg. $c$ and $h$ are the velocity of light and the Planck's constant respectively. The photo ionization code (cloudy 90) was run for a range of hydrogen column densities and a table $\frac{f_{\text {ion }}}{Z}$ vs. $\Gamma$ was prepared for each value of hydrogen column density. These values are quite insensitive to the values of particle densities and abundances. This table was used to determine the value of $\frac{f_{\text {ion }}}{Z}$ for any value of $\Gamma$ and $N_{\mathrm{H}}$ during the calculations.

The column density distribution function for a particular ion produced by halos with circular velocity $V_{1}<V_{\mathrm{C}}<$ $V_{\mathrm{u}}$ is given as,

$$
\begin{aligned}
& \frac{\mathrm{d}^{2} N\left(z, N_{\text {ion }}\right)}{\mathrm{d} N_{\text {ion }} \mathrm{d} z} \\
& =\frac{c}{H_{0}}(1+z)^{1 / 2} \int_{V_{1}}^{V_{\mathrm{u}}} n\left(V_{\mathrm{c}}, z\right) \epsilon \mathrm{d} V_{\mathrm{c}} \int_{0}^{1} \mathrm{~d} \mu\left|\frac{\partial A\left(N_{\text {ion }}, \mu\right)}{\partial N_{\text {ion }}}\right| \\
& =\frac{c}{H_{0}}(1+z)^{1 / 2} \int_{V_{1}}^{V_{\mathrm{u}}} n\left(V_{\mathrm{c}}, z\right) \epsilon \mathrm{d} V_{\mathrm{c}} \int_{0}^{1} \mathrm{~d} \mu \frac{\partial}{\partial N} \int_{\theta=0}^{2 \pi} \mathrm{d} \theta \frac{1}{2} P^{2}(\theta, \mu),(3.5)
\end{aligned}
$$

where $\left|\frac{\partial A\left(N_{\mathrm{ion}}, \mu\right)}{\partial N_{\mathrm{ion}}}\right|=\frac{A\left(N_{\mathrm{ion}}+\Delta N_{\mathrm{ion}}, \mu\right)-A\left(N_{\mathrm{ion}}, \mu\right)}{\Delta N_{\mathrm{ion}}}$ and area $A\left(N_{\text {ion }}, \mu\right)=\int_{\theta=0}^{2 \pi} \mathrm{d} \theta \frac{1}{2} P^{2}(\theta, \mu)$ is the projected area, for a given value of $i$, inside which the column density is $>N_{\text {ion }}$ and $n\left(V_{\mathrm{c}}, z\right) \mathrm{d} V_{\mathrm{c}}$ is the number of halos per unit volume with circular velocity between $V_{\mathrm{c}}$ and $V_{\mathrm{c}}+\mathrm{d} V_{\mathrm{c}}$ at redshift $z$. The deceleration parameter $q_{0}$ is taken to be 0.5 . We have chosen flat models for the universe $\left(q_{0}=0.5\right)$ which are popular (Ostriker \& Steinhardt 1995) as they can be produced naturally in any inflationary scenario and require no fine tuning of parameters. These models are also consistent with independent constraints from various cosmological observations. $\epsilon$ is the fraction of halos that give rise to absorption lines; for the galactic halos it is the fraction of halos having sufficient gas to produce absorption lines. For these, $\epsilon$ is taken to be 0.69 , which is the fraction of late-type galaxies (Postman \& Geller 1984). For mini-halos $\epsilon=1$. The redshift distribution of lines of a particular ion with column density greater than $N_{\text {ion }}$ is given by

$$
\begin{aligned}
\frac{\mathrm{d} N\left(z, N_{\text {ion }}\right)}{\mathrm{d} z}= & \frac{c}{H_{0}}(1+z)^{1 / 2} \int_{V_{1}}^{V_{\mathrm{u}}} n\left(V_{\mathrm{c}}, z\right) \\
& \times \int_{0}^{1} \mathrm{~d} \mu A\left(N_{\text {ion }}, \mu\right) \epsilon \mathrm{d} V_{\mathrm{c}} .
\end{aligned}
$$

We assume two models for the velocity distribution of halos:

(i) Mass condensations in the CDM universe obtained using the Press-Schechter theory (hereafter PS distribution): from the CDM models of structure formation (Mo et al. 1993) the mass and circular velocity of a halo are related to the comoving radius $r_{0}$ and redshift $z$ by,

$M=\frac{4 \pi}{3} \rho_{0} r_{0}^{3}, \quad V_{\mathrm{c}}=1.67(1+z)^{1 / 2} H_{0} r_{0}$,

where $\rho_{0}$ is the mean density of the universe at the present time. The number of halos per unit volume with circular velocity between $V_{\mathrm{c}}$ and $V_{\mathrm{c}}+\mathrm{d} V_{\mathrm{c}}$ is given by

$$
\begin{aligned}
n\left(V_{\mathrm{c}}, z\right) \mathrm{d} V_{\mathrm{c}}= & \frac{-3(1.67)^{3} \delta_{c} H_{0}^{3}(1+z)^{5 / 2}}{(2 \pi)^{3 / 2} V_{\mathrm{c}}^{4} \Delta\left(r_{0}\right)} \frac{\mathrm{d} \ln \Delta}{\mathrm{d} \ln V_{\mathrm{c}}} \\
& \times \exp \left(\frac{-\delta_{\mathrm{c}}^{2}(1+z)^{2}}{2 \Delta^{2}\left(r_{0}\right)}\right) \mathrm{d} V_{\mathrm{c}}
\end{aligned}
$$

Here $\delta_{\mathrm{c}}=1.68$ and the functional form of $\Delta\left(r_{0}\right)$ for the CDM power spectrum of density perturbation is

$$
\Delta\left(r_{0}\right)=16.3 b_{\mathrm{g}}^{-1}\left(1-0.3909 r_{0}^{0.1}+0.4814 r_{0}^{0.2}\right)^{-10},
$$

where $b_{\mathrm{g}}$ is the bias parameter, which is taken to be 1 in our calculations.

(ii) Observed (Schechter) luminosity function of galaxies (hereafter LF distribution): the number of spiral galaxies per unit volume, with luminosity between $L$ and $L+\mathrm{d} L$, is given by,

$n(L) \mathrm{d} L=\phi^{*}\left(\frac{L}{L_{*}}\right)^{\alpha} \exp \left(-\frac{L}{L_{*}}\right) \mathrm{d} L$.

Here $\phi^{*}$ and $\alpha$ give the normalization and the index of faint end slope of the luminosity distribution. $L^{*}$ is the luminosity of the galaxies having circular velocity $V_{\mathrm{c}}^{*}$ which we take as $250 \mathrm{~km} \mathrm{~s}^{-1}$. The luminosity $L$ is related to circular velocity $V_{\mathrm{c}}$ as $\frac{L}{L_{*}}=\left(\frac{V_{\mathrm{c}}}{V_{\mathrm{c}}^{*}}\right)^{2.6}$ for spiral galaxies. Bromley et al. (1998) have determined the values of the luminosity function parameters for six spectral type of galaxies in two different environments. These values are used here to calculate the total number of galaxies as a function of the circular velocity and redshift.

It is possible that the number density of galaxies has been higher in the past and reduced with time due to merger of galaxies. We also consider this possibility. The number density of galaxies after merging is given by $n(\delta t)=f(\delta t) n_{0}$ (Park \& Gott 1997) where $n_{0}$ is the present number density of galaxies and $\delta t$ is the look back time of the universe given by, $\delta t=\frac{2}{3 H_{0}}\left(1-(1+z)^{-1.5}\right)$. We assume that there is no evolution of the velocity dispersion. The strength and time dependence of merging is described by the function $f(\delta t)$ which is assumed to be $\exp (Q(\delta t))$. The merging rate $Q$ roughly corresponds to the average number of pieces at $z \sim 0.65$ that merged to produce one galaxy at $z=0$. The value of $Q$ varies between 2 to 4 for different models of the universe; here we take $Q=3.5$ as that gives the best match between model predictions and observations. 


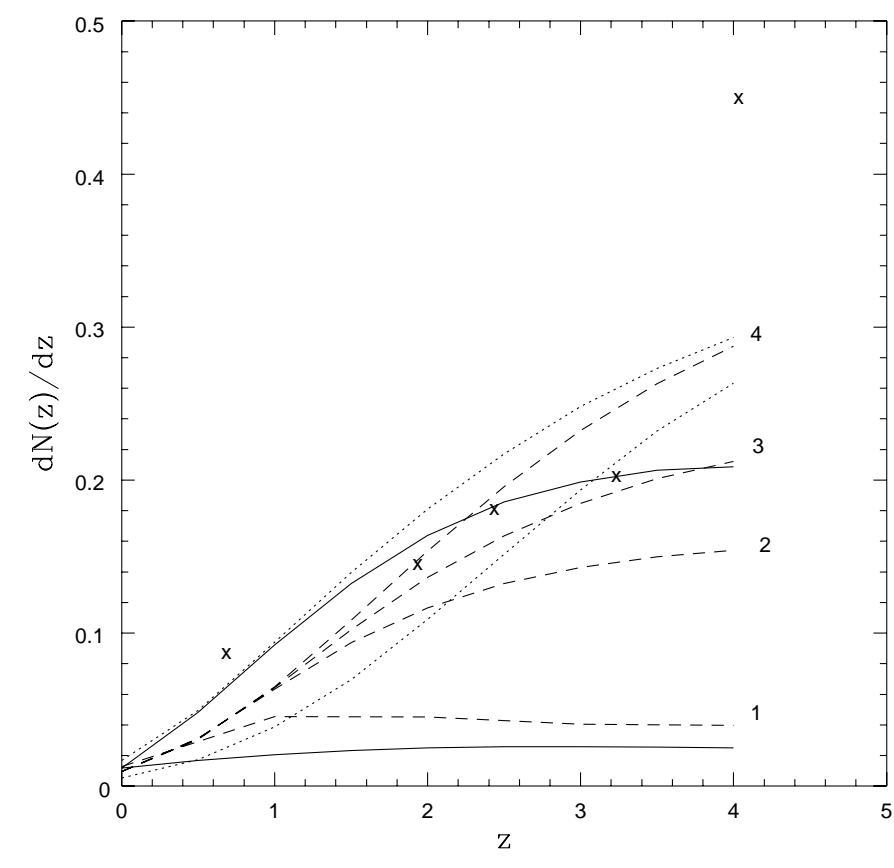

Fig. 1. Redshift distribution of DLASs with column density greater than $10^{20.3} \mathrm{~cm}^{-2}$. Dashed lines marked 1, 2, 3, 4 are for $a=0.0,0.7,0.8,0.9$ respectively for PS distribution. Upper and lower dotted lines are for $t=0.005 R_{\mathrm{d}}$ and $0.02 R_{\mathrm{d}}$ respectively for the PS distribution. All other lines are for $t=0.01 R_{\mathrm{d}}$. Upper and lower solid lines are for LF distribution with and without mergers respectively. Points marked " $\times$ " are observed points from Storrie-Lombardi et al. (1996).

\section{Constraining the model parameters}

The free parameters in our model are $\eta_{\mathrm{cl}}(0)$, the number of clouds per unit volume at the centre, thickness of the disc, $t$ and $a$, which determines the rate of evolution of the disc size. Increasing $t$ decreases the particle density and therefore decreases the fraction of neutral hydrogen. A given value of $N_{\mathrm{HI}}$ is thus produced at smaller values of the impact parameters for larger values of $t$, reducing the number of DLAs per unit redshift. Change in $a$ on the other hand changes the slope of the redshift distribution of the DLAs.

The observed number density of DLA absorbers as a function of redshift has been obtained by Lanzetta et al. (1995) and Storrie-Lombardi et al. (1996). The number density evolution of DLASs with $N_{\mathrm{HI}}>20.3$ for our model is shown in Fig. 1 for 4 values of a and three values of $\mathrm{t}$ for PS distribution and $a=0.9$ and $t=0.01 R_{\mathrm{d}}$ for LF distribution. We find that the model results for the redshift distribution of DLAs match best with the observations for redshifts smaller than 4 for $t \simeq 0.01 R_{\mathrm{d}}$ and $a \simeq 0.9$. These are the values we use in the rest of the calculations. We note, however, that the observed number of DLASs at $z \sim 4$ is much larger than the prediction of all our models. Note that as the DLASs are formed purely by the disc, their number does not depend on $\eta_{\mathrm{cl}}(0)$.

Storrie-Lombardi et al. (1994) have studied the redshift distribution of LLSs and have found it to be roughly

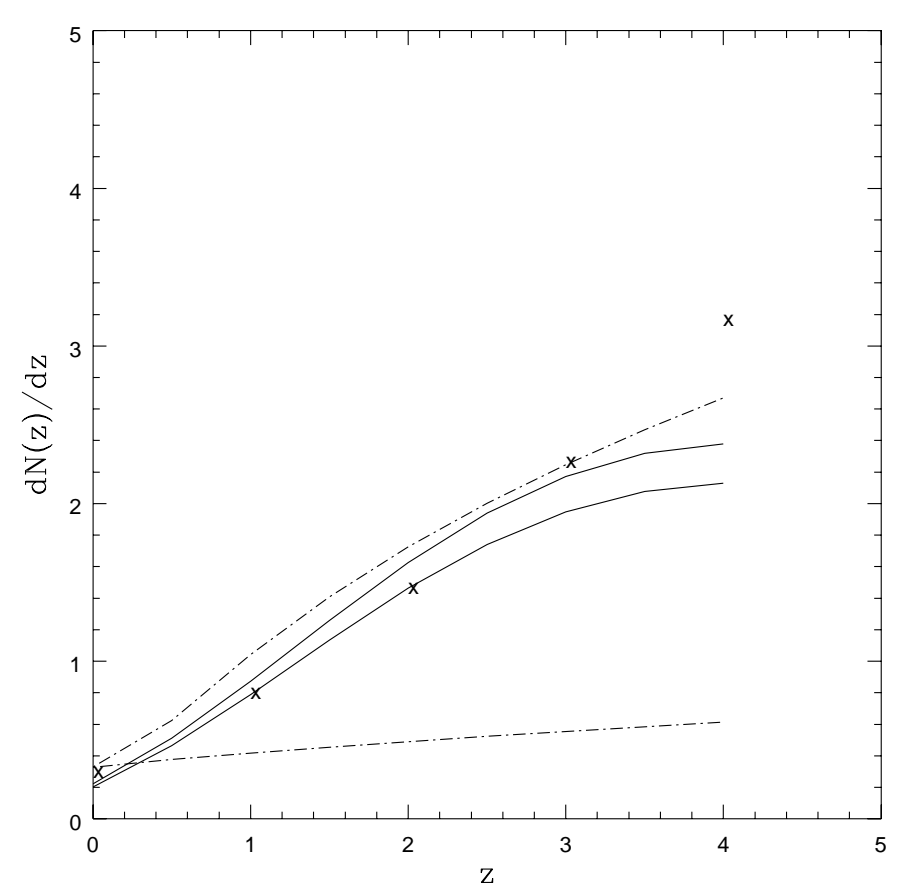

Fig. 2. Redshift distribution of LLSs. Lower and upper solid lines are for the PS distribution for $R_{\mathrm{H}}=500 \mathrm{kpc}$ for $f=$ 0.0030 and 0.0035 respectively. Upper and lower dash-dotted lines are for LF distribution and $R_{\mathrm{H}}=500 \mathrm{kpc}$ with and without mergers respectively with $f=0.0015$. Points marked " $\times$ " are observed points from Storrie-Lombardi et al. (1994).

a power law with slope 1.55 over the redshift range 0.40 to 4.69 . However, they find that the distribution becomes steeper for redshifts $z>2.5$. Their values are plotted in Fig. 2. The figure also shows the results of our calculations for the number density evolution of LLSs for two values of $f$ (which determines the number of clouds per unit volume at the centre of the galaxy, see Eq. (2.7)) and for the halo radius $R_{\mathrm{H}}=500 \mathrm{kpc}$ for PS distribution. The radius of the halo does not make any difference to the distribution. This is because the contribution from the outer halo to HI column density is negligible for los producing LLSs. The value of $f=0.0035$ roughly agrees with the observed values for $z<4$ for PS distribution. The values for $\mathrm{LF}$ distribution are also plotted in the figure for $f=0.0015$ and $R_{\mathrm{H}}=500 \mathrm{kpc}$ which are in reasonable agreement with the observed values. We use $f=0.0035$ for PS distribution and $f=0.0015$ for LF distribution in what follows. Here again we note that for $z \sim 4$, the observed number of LLS is considerably larger than the model predictions. For $f=0.0035$ we estimate that a los with impact parameter $\leq 100 \mathrm{kpc}$ through halos with $V_{\mathrm{c}}>150 \mathrm{~km} \mathrm{~s}^{-1}$ will have a $>90 \%$ probability of intersecting a cloud. The probability is, however, less than $90 \%$ for $f=0.0015$. The value of f obtained for PS distribution is therefore consistent with the lensing data.

The number density evolution of LAASs is displayed in Fig. 3 for $R_{\mathrm{H}}=1000$ and $500 \mathrm{kpc}$. As seen, this is sensitive to the value of halo radius as los producing LAASs does get significant contribution from the outer parts of 


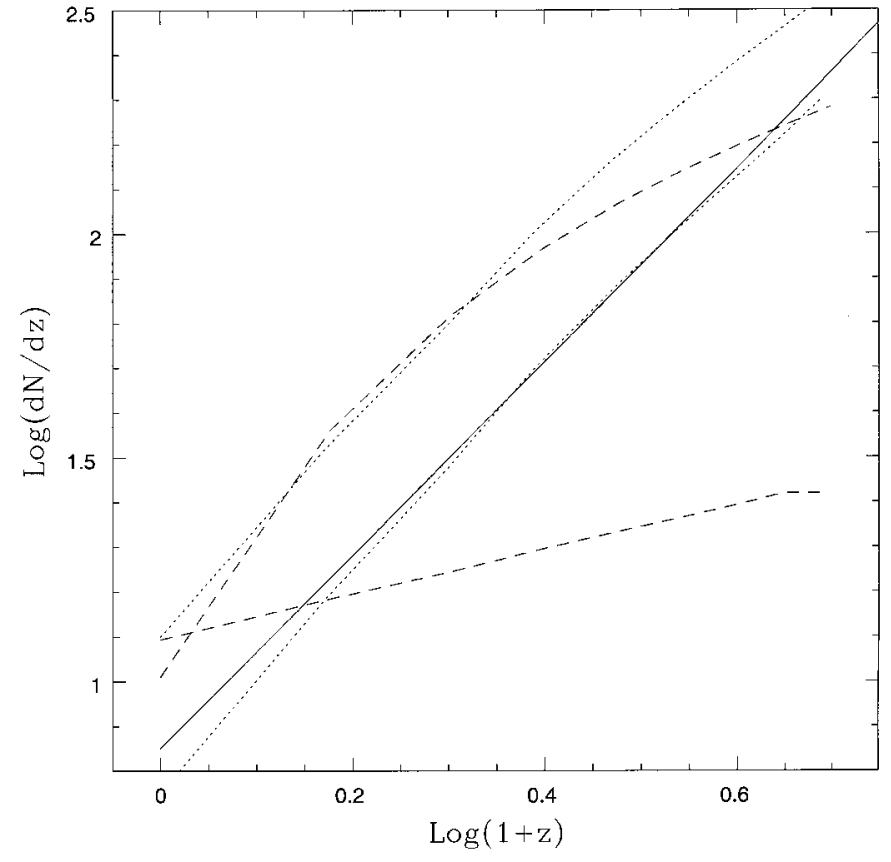

Fig. 3. Redshift distribution of LAASs with column density greater than $10^{13.77} \mathrm{~cm}^{-2}$. The solid line is the observed distribution taken from Kim et al. (1998). Upper and lower dotted lines are for $R_{\mathrm{H}}=1000$ and $500 \mathrm{kpc}$ respectively with $f=0.0035$ for PS distribution. Upper and lower dashed lines are for $R_{\mathrm{H}}=500$ for LF distribution with and without mergers respectively with $f=0.0015$.

the halo. The figure also shows the observed redshift distribution (taken from Kim et al. 1997) for column density $>10^{13.77} \mathrm{~cm}^{-2}$, which is also the lower limit used for the theoretical curves. The distribution is consistent with the observed distribution for $R_{\mathrm{H}}=500 \mathrm{kpc}$, for both PS and LF distribution. We, note, however, that the contribution of the minihalos is independent of the value of their radius as long as it is larger than $250 \mathrm{kpc}$. So we have assumed the minihalo radius to be $250 \mathrm{kpc}$ and the galactic halo radius to be $500 \mathrm{kpc}$ in the rest of the calculations.

It can be seen from Figs. 1, 2 and 3 that the use of LF distribution without merger is inconsistent with the observations of the redshift evolution of DLASs, LLSs and LAASs. The observations thus do indicate a strong evolution in the comoving number density of galaxies.

The column density distribution function for hydrogen, $f\left(N_{\mathrm{HI}}\right)$, is defined as the number of absorbing systems per unit column density per unit redshift path which is defined by $X(z)=\frac{2}{3}\left[(1+z)^{3 / 2}-1\right]$ for $q_{0}=0.5$. A comparison of the observed column density distribution with the predictions of our model is presented in Fig. 4 for the column density range $10^{12}-10^{22} \mathrm{~cm}^{-2}$ at redshift $z=2.5$. The observed data are from Storrie-Lombardi et al. (2000). A reasonably good match between the theoretical and observed distributions is obtained for both PS and LF distribution.

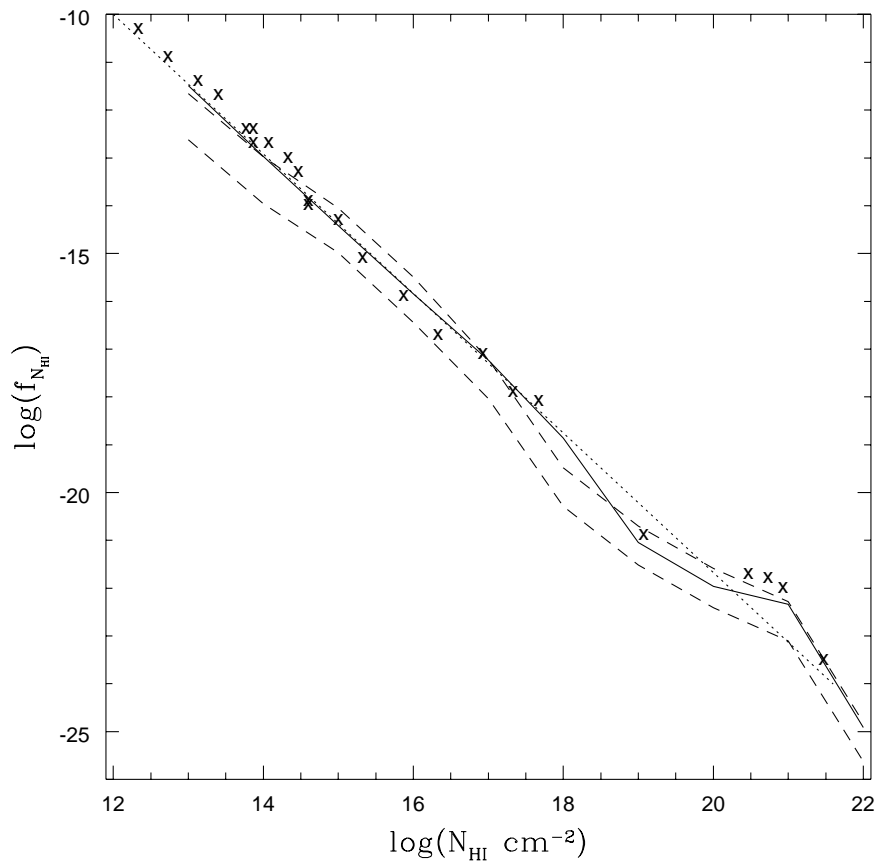

Fig. 4. Column density distribution of HI lines at $z=2.5$. Points marked " $\times$ " are observed data taken from StorrieLombardi et al. (2000) and the dotted line is the best fit to the points. The solid line is for PS distribution with $a=0.9$, $f=0.0035, R_{\mathrm{H}}=500 \mathrm{kpc}$. Upper and lower dashed lines are for LF distribution with and without mergers respectively with $a=0.9, f=0.0015, R_{\mathrm{H}}=500 \mathrm{kpc}$.

\section{Redshift distribution of heavy element absorption systems}

For determining the redshift distribution of HEASs, one has to consider the change in abundance of heavy elements with redshift. It seems possible that the abundance in galactic halos has been increasing continuously with time because of in situ star formation (Khare \& Rana 1993; Chiba \& Nath 1997). We therefore assume the abundance of heavy elements to depend on redshift as $Z(z)=Z(0)(1+z)^{-\delta}$ in the galactic halos as well as galactic discs and consider several values for $\delta$. We have also considered another case of an assumed abundance gradient $Z(r, z)=Z(0, z) \mathrm{e}^{-2 r / V_{\mathrm{c}}}$ for the heavy elements produced in situ in the galaxies. As there is no star formation in the minihalos, the abundance in these halos has been assumed to be $[\mathrm{Z} / \mathrm{H}]=-2.5$, which has been observed in low column density Ly alpha clouds (Songaila \& Cowie 1996).

The redshift evolution of CIV for $N_{\mathrm{CIV}}>10^{13} \mathrm{~cm}^{-2}$ is shown in Fig. 5 for $\delta=0$ and 3 and for $\delta=3,4$ with an assumed abundance gradient for PS distribution. For LF distribution lines are shown for $\delta=0$ and 3 with an abundance gradient without merger and $\delta=3$ and 4 with an abundance gradient with merger. In order to obtain the value of $\mathrm{d} N / \mathrm{d} z$ from observations, a maximum likelihood analysis was performed with the data collected from the literature (references given in Das \& Khare 1999). The data include components of DLASs also. These are 


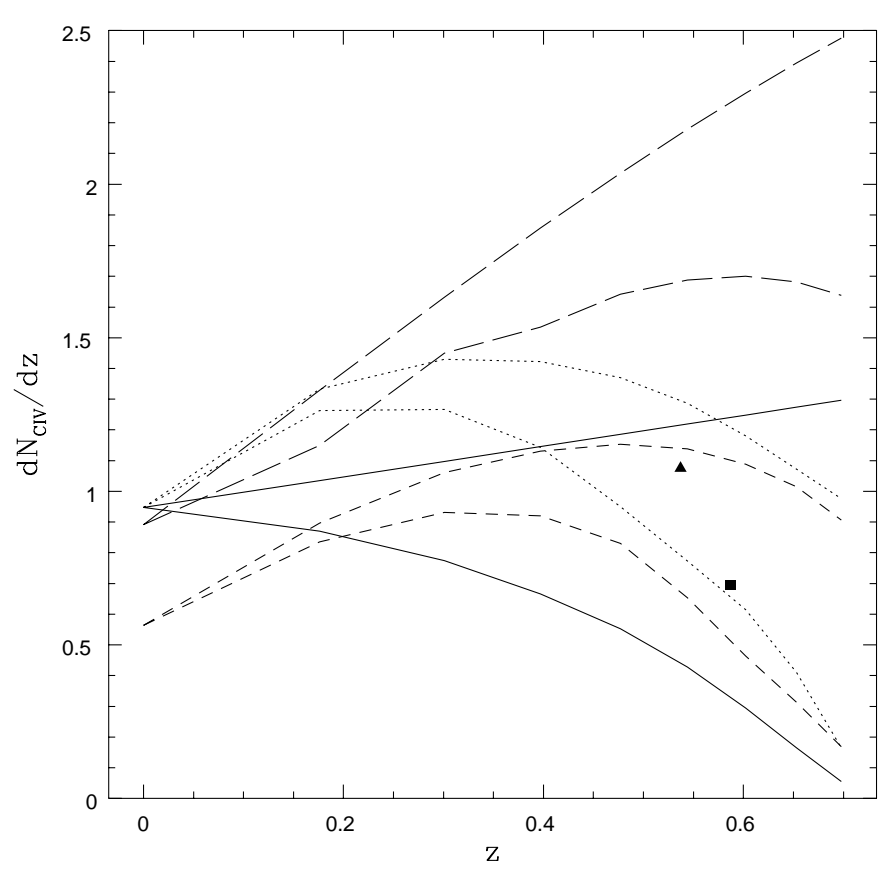

Fig. 5. Redshift distribution for C IV lines with $N_{\mathrm{CIV}}>$ $10^{13.0} \mathrm{~cm}^{-2}$. Upper and lower long dashed lines are for $\delta=0$ and 3 respectively for PS distribution. Upper and lower short dashed lines are for $\delta=3$ and 4 with an abundance gradient respectively for PS distribution. Upper and lower dotted lines are for $\delta=3$ and 4 with an abundance gradient respectively for LF distribution with mergers. Upper and lower solid lines are for $\delta=0$ and 3 with an abundance gradient respectively for LF distribution without mergers. The triangle shows the observed value for the data set collected from the literature as described in the text. and the square represents the value for the data from Songaila (1998).

included in the data, since most of the components are likely to arise in the galactic halos. For this analysis we have only used the absorption systems having a relative velocity w.r.t. the QSOs larger than $5000 \mathrm{~km} \mathrm{~s}^{-1}$. We have thus excluded any intrinsic absorption systems that may be present in the QSO spectra. The same holds for any other observational data used in this paper. The observed values are plotted in Fig. 5. Songaila (1998) has reported observations of CIV toward 13 QSOs. The value of $\mathrm{d} N / \mathrm{d} z$ calculated for her data by maximum likelihood analysis is also plotted in the figure. As seen from the figure, high $\delta$ values $(3,4)$ are indicated by the observations.

We have constructed a sample of observed MgII column densities in individual components in the absorption systems at redshift $z \sim 0.5$ from the literature. We have thus included all the observations of Lanzetta \& Bowen (1992); Carswell et al. (1991) and Petitjean \& Bergeron (1990) and performed a maximum likelihood analysis. The resulting number of systems per unit redshift for lines with $N_{\text {MgII }}>10^{13.15} \mathrm{~cm}^{-2}$ seems to require $\delta \geq 0$. However, the observed value is for $z \simeq 1$. More data at higher redshifts are required to draw any firm conclusion about the value of $\delta$.

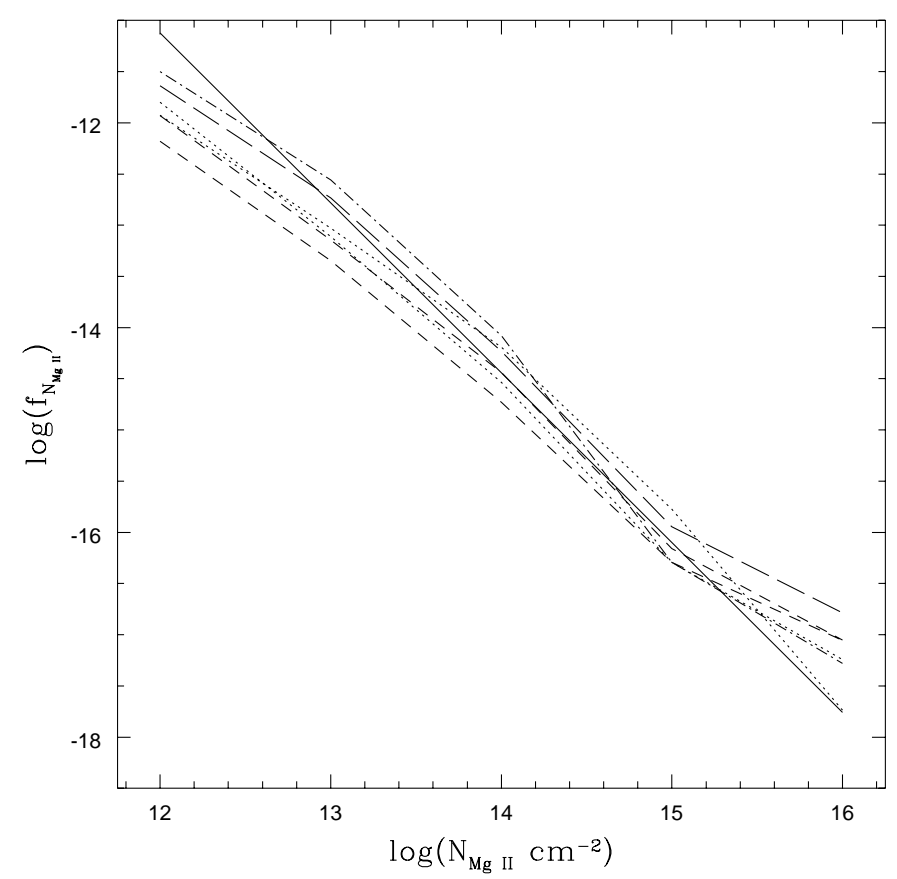

Fig. 6. Column density distribution for MgII lines at $z=0.5$. Solid line is the observed distribution which is the result of the maximum likelihood analysis of the data taken from the literature. Upper and lower dashed lines are for $\delta=3$ and $\delta=3$ with abundance gradient respectively for PS distribution. Upper and lower dotted lines are for $\delta=0$ and 3 respectively for LF distribution without mergers. Long dashed and dash dotted lines are for $\delta=0$ and 3 respectively for LF distribution with mergers.

\section{Column density distribution of heavy element lines}

The density distribution function for $\mathrm{MgII}, f_{N_{\mathrm{MgII}}}$, is shown in Fig. 6, for the two velocity distribution of the absorbers. Observed values which are the result of maximum likelihood analysis of the data sample collected from Lanzetta \& Bowen (1992), Carswell et al. (1991) and Petitjean \& Bergeron (1990) are also plotted in the figure. As evident from the figure, there is a reasonably good agreement of the model results with the observations with $\delta=3$. However, the column density distribution, being plotted on a logarithmic scale, is not very sensitive to the delta values used.

The column density distribution of CIV lines for $2.52<$ $z<3.78$ has recently been obtained from high resolution Keck observations by Songaila (1998). This distribution is roughly a power law with a slope of -1.5 for column densities larger than $6 \times 10^{12} \mathrm{~cm}^{-2}$ for which her sample is complete. We have plotted model results for the column density distribution at $z=3$ for several $\delta$ values in Fig. 7 for both PS and LF distribution. Here, as in the case of redshift evolution, results for high $\delta$ values i.e. 3 and 4 are in better agreement with the observations. However, at lower column densities, the model predicts many more lines than the observed number. The observed data, however, may be incomplete below $6 \times 10^{12} \mathrm{~cm}^{-2}$ as noted by 


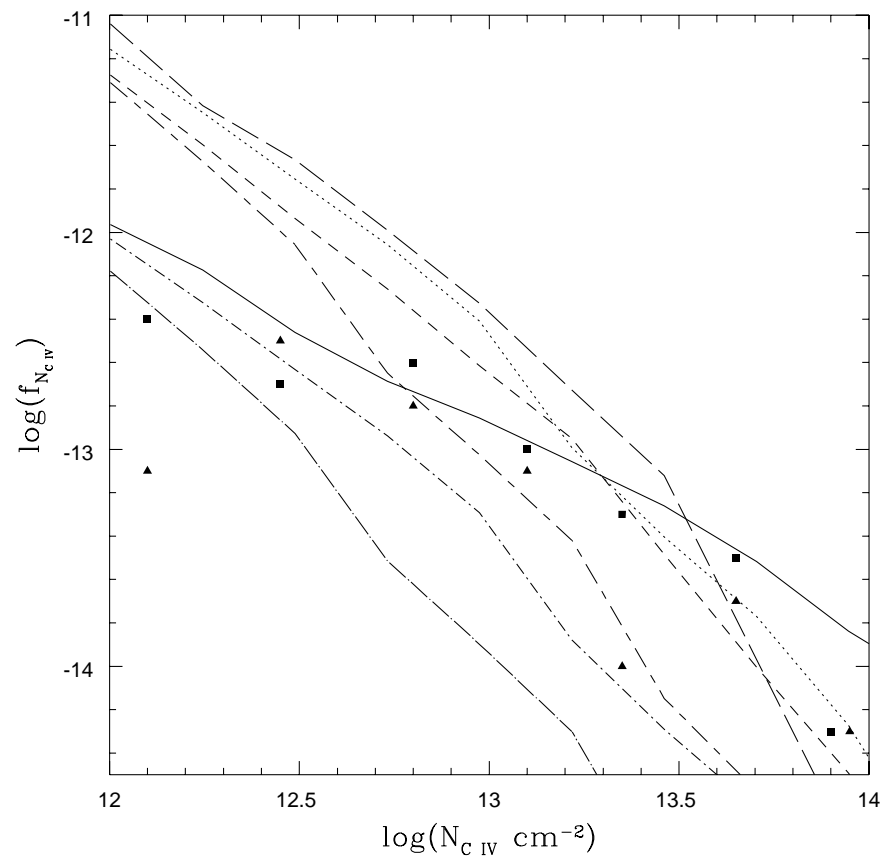

Fig. 7. Column density distribution for C IV lines at $z=3.0$. Long-dashed line is for $\delta=4$ and short-dashed line is for $\delta=3$ with an abundance gradient for PS distribution. Dotted and long-short dashed line are for $\delta=3$ and 4 respectively with an abundance gradient for LF distribution with mergers. Solid line is for $\delta=0$ for LF distribution without mergers. Short dash-dotted and long dash dotted lines are for $\delta=3,4$ respectively with an abundance gradient for LF distribution without mergers. Triangles and squares are the observed values from Songaila (1998) for $z<3$ and $z \geq 3$ respectively.

Songaila (1998) and it is possible that the number of small column density lines may actually be considerably larger. An increase in the rate of abundance evolution does not serve the purpose, since it reduces the number of higher column density lines more than that of lower column density lines.

\section{Conclusion}

Pure halo or pure disc will not produce sufficient variety in the properties of the QSO absorption lines to explain the ensemble of observed lines. To better understand the structure of the absorbers and to set constraints on the their properties, we have presented a model which includes minihalo, galactic halos and galactic discs. We compared various observed distributions of the absorption lines with the predictions from our model.

The main result of the work presented in this paper is that the statistical distributions of various classes of absorption systems can be reasonably well explained by the three component models which are expected to arise naturally in CDM cosmology. We do not claim the model to be unique and there could very well be other sets of parameters which could also explain the observations equally well. We also note that some of the statistical properties, like the redshift distribution of $\mathrm{MgII}$ lines are not very well determined and may change when more observations become available. However at present we find the observations to be consistent with the CDM cosmology.

Some of more quantitative results are as follows.

(1) The ratio of the disc radius to virial radius decreases with time roughly as $(1+z)^{0.9}$.

(2) For our assumed baryonic mass of the halo clouds $\left(\sim 10^{6} M_{\odot}\right)$ there are roughly up to a few hundred clouds in the halos. Thus roughly a few percent of the baryonic halo mass is in the form of clouds.

(3) The Lyman alpha lines with column density $>10^{13.77} \mathrm{~cm}^{-2}$ seem to require the radius of the galactic halos to be around $500 \mathrm{kpc}$, assuming this radius to be independent of redshift.

(4) The column density distribution of $\mathrm{HI}$ and MgII is rather insensitive to variation in various parameters and is in an excellent agreement with most models. The agreement of the observed column density distribution of HI with the model results for over 8 orders of magnitude in column density does strengthen the likelihood of a unified model like the one presented in this paper.

(5) The redshift distribution and column density distribution of CIV lines does require strong metallicity evolution with redshift as $(1+z)^{-\delta}, \delta$ being $\geq 3$, along with an assumption of the presence of an abundance gradient in the halo, the abundance decreasing exponentially with distance. However, we note that CIV lines mostly arise in clouds embedded in the halos and give us information regarding the abundance in the halo and not in the discs. The observations of MgII lines which get contributions from the disc also are not sufficiently well known to give direct evidence for or against such an abundance evolution in the discs.

(6) The observed luminosity function of galaxies is consistent with the observations of QSO absorption lines only if one assumes that the comoving number density of galaxies was larger in the past and reduced with time due to merging.

\section{Discussion}

We have presented above a unified model for QSO absorbers where various classes of QSO absorption systems are produced (apart from the few low column density Ly $\alpha$ lines produced by the minihalos) by lines of sight crossing spiral galaxies at different values of impact parameters. As seen above the model is able to reproduce the observed redshift and column density distributions of various species of the absorption systems reasonably well. Several doubts have been expressed over such a unified scheme in the literature. Bowen et al. (1995) noticed considerable effect of environment on the absorption lines produced by galaxies. They, therefore cautioned against constructing models of generic galactic halos. Diverse morphologies have also been observed for DLA galaxies (Le Brun et al. 1997; Turnshek et al. 2000; Kulkarni et al. 2000). 
LLS are known to originate in bright galaxies (Steidel et al. 1995). However, it has been suggested lately that DLASs are produced by low surface brightness galaxies. High redshift DLASs have not been detected in Ly $\alpha$ or $\mathrm{H} \alpha$ emission except in a few cases, while low redshift DLASs have been observed to be associated with low surface brightness galaxies (Turnshek et al. 2000; Pettini et al. 2000). Rao \& Turnshek (2000) found no evolution in the HI content of DLASs over the redshift range of 4.0 to 1.0. As a lot of star formation has been presumably taking place in the bright spiral galaxies over this period it would have led to a significant decrease in HI content of these galaxies. They therefore suggest that DLASs and star forming galaxies may be different populations. Pettini et al. (1999) showed that DLASs do not show significant chemical evolution from $z \simeq 3$ to 0.3 . They also therefore suggest the DLASs to be different from bright galaxies which undergo extensive star formation and evolution. They suggest that the bright galaxies are under represented in DLA samples precisely for this reason. The bright galaxies having higher metallicity and therefore higher dust content will render the QSOs extinct. Savaglio (2000) noted that the metallicity discussed by Pettini et al. (1999) (being weighted by column density) is dominated by high column density systems which may not be uniformly distributed over the entire redshift range. Restricting to a sample of 50 low column density $\left(N_{\mathrm{HI}}<10^{20.8} \mathrm{~cm}^{-2}\right)$ DLASs which are presumably dust free she found evidence for chemical evolution. The total sample of 75 QSOs also shows evolution if dust depletion is corrected for. She found the global metallicity to change from $Z_{\odot} / 30$ to $Z_{\odot}$ from $z \sim 4.1$ to 0.4 . It is therefore possible that the observed DLA sample is biased towards LSB galaxies.

Theoretical studies (Mo et al. 1998; Mo \& Mao 1999) show that the halo spin parameter plays an important role in determining the properties of galaxies forming within halos of dark matter. Halos of small angular momentum give rise to compact high density systems while halos of low mass and high angular momentum form discs with a low surface density of baryons. The former are sites of star formation while the later dominate the absorption cross section.

Our model does not take into account the presence of dust and assumes the chemical abundance to be independent of the mass of the galaxies at a given redshift, though in some of the models we do assume the chemical abundance to decrease with radial distance, the rate of decrease being higher for halos with lower circular velocities. However, even if high metallicity systems are indeed under represented in the observed DLA sample the rate of chemical evolution deduced from our model will not change. This is because the rate of chemical evolution obtained here is from the redshift evolution of the CIV lines with column density $\geq 10^{13} \mathrm{~cm}^{-2}$ which is decided by the los passing only through the halo. Thus the chemical evolution deduced in our models is confined to the galactic halos and does not necessarily reflect a corresponding chemical evolution in the discs. The thickness of the discs may, however, have to be decreased further in order to account for the DLASs which are unobserved due to dust extinction.

Acknowledgements. The authors wish to thank the referee, Joe Wampler, for his numerous suggestions which improved the manuscript considerably. This work was partially supported by a grant (No. SP/S2/013/93) by the Department of Science and Technology, Government of India. S.D. and A.S. are supported by C.S.I.R. fellowships.

\section{References}

Barnes, J., \& Efstathiou, G. P. 1987, ApJ, 319, 575

Bergeron, J. 1988, in QSO absorption lines: Probing the universe, ed. J. C. Blades, D. A. Turnshek, \& C. A. Norman (Cambridge University Press), 147

Bromley, B. C., Press, W. H., Lin, H., \& Kirshner, R. P. 1998, ApJ, 505, 25

Bowen, D. V., Blades, J. C., \& Pettini, M. 1995, ApJ, 448, 662

Carswell, R. F., Lanzetta, K. M., Parnell, H. C., \& Webb, J. K. 1991, ApJ, 371, 36

Charlton, J. C., \& Churchill, C. W. 1996, ApJ, 465, 631

Charlton, J. C., Edwin, E. S., \& Craig, J. H. 1993, ApJ, 402, 493

Chen Hsiao-Wen, Lanzetta, K. M., Webb, J. K., \& Barcons, X. 1998, ApJ, 498, 77

Chen Hsiao-Wen, Lanzetta, K. M., \& Fernandez-Soto, A. 2000, ApJ, 533, 120

Chiba, M., \& Nath, B. B. 1997, ApJ, 483, 638

Cowie, L. L., Songaila, A., Kim, T., \& Hu, E. M. 1995, AJ, 109, 1522

Das, S., \& Khare, P. 1999, ApJ, 510, 597

Gnedin, N. Y., \& Ostriker, J. P. 1997, ApJ, 486, 581

Jedamzik, K., \& Prochaska, J. X. 1998, MNRAS, 296, 430

Kauffmann, G. 1996, MNRAS, 281, 475

Kennicutt, R. C. 1989, ApJ, 344, 685

Khare, P., \& Rana, N. C. 1993, JApA, 14, 83

Kim, T., Hu, E. M., Cowie, L. L., \& Songaila, A. 1997, AJ, 114,1

Kulkarni, V. P., Hill, J. M., Schneider, G., et al. 2000, ApJ, 536,36

Lanzetta, K. M., \& Bowen, D. V. 1992, ApJ, 391, 48

Lanzetta, K. M., Bowen, D. V., Tytler, D., \& Webb, J. K. 1995, ApJ, 442, 538

Le Brun, V., Bergeron, J., Boisse, P., \& Deharveng, J. M. 1997, A\&A, 321, 733

Mo, H. J., \& Mao, S. 2000, MNRAS, 318, 163

Mo, H. J., Miralda-Escude, J., \& Rees, M. J. 1993, MNRAS, 264, 705

Mo, H. J., Mao, S., \& White, S. D. M. 1998, MNRAS, 295, 319

Ostriker, J. P., \& Steinhardt, P. J. 1995, Nature, 377, 600

Park, M., \& Gott, III J. R. 1997, ApJ, 489, 476

Petitjean, P., \& Bergeron, J. 1990, A\&A, 231, 309

Pettini, M., Ellison, S. L., Steidel, C. C., \& Bowen, D. V. 1999, ApJ, 510, 576

Pettini, M., Ellison, S. L., Steidel, C. C., et al. 2000, ApJ, 532,65

Postman, M., \& Geller, M. J. 1984, ApJ, 281, 95

Prochaska, J. X., \& Wolfe, A. M. 1997, ApJ, 474, 140 
Rao, S. M., \& Turnshek, D. A. 2000, ApJS, 130, 1

Sargent, W. L. W., Young, P. J., Boksenberg, A., \& Tytler, D. 1980, ApJS, 42, 41

Savaglio, S. 2000, IAUS, 204, 245

Smette, A. 1995, in QSO Absorption Lines, ed. G. Meylan (Springer), 276

Songaila, A. 1998, AJ, 115, 218

Songaila, A., \& Cowie, L. L. 1996, AJ, 112, 335

Srianand, R., \& Khare, P. 1994, MNRAS, 271, 81

Steidel, C. C. 1993, in Galaxy Evolution: The Milky Way perspective, ed. S. R. Majewski, ASP, Conf. Ser., 49, 227
Steidel, C. C., Bowen, D. V., Blades, J. C., \& Dickenson, M. 1995, ApJ, 440, 45

Storrie-Lombardi, L. J., \& Wolfe, A. M. 2000, ApJ, 543, 552

Storrie-Lombardi, L. J., McMahon, R. G., Irwin, M. J., \& Hazard, C. 1994, ApJ, 427, L13

Storrie-Lombardi, L. J., Irwin, M. J., \& McMahon, R. G. 1996, MNRAS, 282, 1330

Turnshek, D., Rao, S., Lane, W., et al. 2000 [astro-ph/0009096]

Wolfe, A. M., Turnshek, D. A., Smith, H. E., \& Cohen, R. D. 1986, ApJS, 61, 249 